


\title{
Resonance Localization in Tokamaks Excited with ICRF Waves *
}

\author{
G. D. Kerbel and M. G. McCoy \\ Natioral Magnetic Fusion Energy Computer Center \\ Lawrence Livermore National Laboratory
}

Advanced wave models used to evaluate ICRH in tokamaks typically use warm plasma theory and allow inhomogeneity in one dimension. The majority of these calculations neglect the fact that gyrocenters experience the inhomogeneity via their motion parallel to the magnetic field. The non-local effects of rotational transform and toroidicity can play a significant role in both the propagation and the absorption physics. In strongly driven systems, wave damping can distort the particle distribution function supporting the wave and this produces changes in the absorption. The most common approach is to use Maxwellian absorption rates. We have developed a bounce-averaged Fokker-Planck quasilinear computational model which evolves the population of particles on more realistic orbits. Each wave-particle resonance has its own specific interaction amplitude within any given volume element: these data need only be generated once. and appropriately stored for efficient retrieval. The wave-particle resonant interaction then serves as a mechanism by which the diffusion of particle populations can proceed among neighboring orbits. The local specific spectral energy absorption rate is directly calculable once the orbit geometry and populations are determined. The code is constructed in such fashion as to accomodate wave propagation models which provide the wave spectral energy density on a poloidal cross-section. Information provided by the calculation includes the local absorption properties of the medium which can then be exploited to evolve the wave field.

\section{RF Power Absorption}

The RF power absorbed on a flux surface per unit toroidal extent by resonant particles can be represented in terms of the quasilinear component of the Fokker-Planck operator:

$$
\boldsymbol{P}_{R F}=\int d^{3} v_{0} \frac{1}{2} m v_{0}^{2} \frac{\partial \lambda \mathcal{F}_{0}}{\partial t}=-\int d^{3} v_{0} \frac{1}{2} m v_{0}^{2} \nabla_{\mathbf{v}_{0}} \cdot \widehat{\Gamma}_{0_{q l}} .
$$

where $\lambda=v_{0} \cos \theta_{0} \tau_{B}$ and all quantities are represented in the constants of motion (velocity) space $\left(v_{0}, \theta_{0}\right)$. After an integration by parts. the RF power absorbed can be cast in the form

$$
P_{R F}=-2 \pi \int d v_{0} \int d \theta_{0} m v_{0} \sin \theta_{0} B_{0_{q l}} \widehat{\Delta}_{0} \mathcal{J}_{0}
$$

where the differential operator $\widehat{\Delta}_{0}$ is given by

$$
\widehat{\Delta}_{0}=\frac{\partial}{\partial v_{0}}+\frac{m \Omega_{0} / \omega-\sin ^{2} \theta_{0}}{v_{0} \sin \theta_{0} \cos \theta_{0}} \frac{\partial}{\partial \theta_{0}}
$$

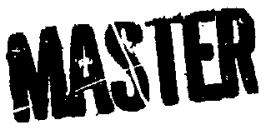

* Work performed under the auspices of the U.S.D.O.E. by LLNL under contract No. W7405-ENG-48. 
and the bounce-averaged quasilinear coefficient $B_{0_{q l}}$ can be expressed in the form

$$
B_{0_{q} t}=-\lambda \frac{q^{2}}{4 m^{2}} \sum_{\text {rays }} \sum_{n}\left\{\left\langle\left\langle\mathbf{E}_{\mathbf{k}} \cdot \Theta_{n} v^{2} \sin \theta e^{i \Psi}\right\rangle\right\rangle^{\star} \Upsilon_{\tau_{B}}\left\langle\left\langle\mathbf{E}_{\mathbf{k}} \cdot \theta_{n} \sin \theta e^{i \Psi}\right\rangle\right\rangle+c c .\right\}
$$

The quantity $\mathbf{E}_{\mathbf{k}} \cdot \boldsymbol{\theta}_{\mathbf{n}}$ signifying the amplitude of the wave-gyrocenter interaction along the unperturbed gyrocenter orbit is viewed as a slowly yarying quantity in the sense that its time variation scales with the bounce time. The eikonal $\Psi$ given by

$$
\Psi(n, t)=\int_{0}^{t} d \tau\left(n \Omega^{\prime}+k_{\|}^{\prime} v_{\|}^{\prime}-\omega\right)=\int_{0}^{t} d \tau \nu(n, \tau)
$$

represents the advance of the interaction phase, $\nu$. along a given gyrocenter trajectory in a single bounce. In large gyration frequency theory. $\Omega_{0} \tau_{B} \gg 1, \Psi$ is viewed as rapidly varying. its time variation scaling with the gyroperiod. The quantity referred to as $\Upsilon$, which accounts for multiple bounce coherence effects, is generally quite close to unity.

The bounce-averages in (2). denoted by $\langle\langle\rangle\rangle$. may be evaluated by the method of stationary phase due to the rapid variation with $\tau$ of $\Psi$. The eikonal $\Psi$ is expanded about the point where $d \Psi / d t$ vanishes (elsewhere the rapid oscillations provide cancelling contributions to the integrand). By (3). this implies

$$
\frac{d \Psi}{d t}=\frac{d}{d t} \int^{t} d \tau \nu(n, \tau)=\nu(n, t)=0
$$

the condition of wave-particle resoirance.

At points for which $d B / d \tau=0$ coincidentally with resonances, not only is $\nu=0$, but also $\dot{\nu}=0$. The expansion of $\Psi$ about these points must be extended to one higher order (including a term in $\ddot{\nu}$ ). We can represent the phase integral in (2) mnemonically by

$$
\int_{0}^{\tau_{B}} d \tau \Pi(n, \tau) e^{i \Psi}=\int_{0}^{\tau_{B}} d \tau \Pi \exp \left(-i \int_{0}^{\tau} d \tau^{\prime}\left(\omega-k_{\|} v_{\|}-n \Omega\right)\left(\tau^{\prime}\right)\right)=\int_{0}^{\theta_{B}} d s \Pi \tau_{c} \delta\left(s-\left.s\right|_{R}\right)
$$

where $\tau_{c}$. which might be called an effective correlation time, is identified through evaluation of the trajectory integral and $\dot{s}_{R}$ is the position at which resonance occurs. Associated with the timelike quantity $\tau_{c}$ through the evaluation of $\Psi$ at the point of stationary phase is a certain phase factor. It is the advance of this phase between subsequent resonances which determines the single bounce coherence of sequential wave-particle interactions. Orbit resonances which are too close to sense significant gyrophase diffusion can interfere constructively or destructively through the action of the relative interaction phase separating them. Details of the caiculation pertaining to this phenomenon are discussed in Kerbel and McCoy ${ }^{1}$.

In reality the wave-particle resonant interaction is not confined to a single point $\left(s_{R}\right)$. but rather occurs over an interval corresponding roughly to the distance the particle (Eyrocenter) moves in a time of the order of $\left|\tau_{c}\right|$. In order to determine the spacial (poloidal) variation of RF power absorption we introduce a resonance broadening model which, apart 
from the normalizing factor $\left|\tau_{c}\right|$. replaces the exponential in the integrand of (5) by a resonance weighting function $w(\tau)$ such that $\int d \tau w(\tau)=1$, the weighting function is normalized; $\int d \tau \tau w(\tau)=\tau_{R}$, the weighting function is centered on the wave-particle resonance: and finally. $\int d \tau\left(\tau-\tau_{R}\right)^{2} w(\tau)=\left|\tau_{c}\right|^{2}$, the width of the weighting function corresponds to the wave-particle correlation time. The stationary phase method alone corresponds to a weighting function with point support. $w(\tau)=\delta\left(\tau-\tau_{R}\right)$.

This resonance broadening technique should be viewed here as a computational convenience rather than a theoretical generalization. It is a physically motivated artifice which permits the wave damping calculated through the quasilinear model to be used concurrently with a wave propagation calculation. We sacrifice no physical insight by neglecting the broadening in the description of the underlying structure of the asymptotic theory.

Figure 1. shows the phaseflow corresponding to the set of velocity space meshpoints chosen to represent the distribution $\mathcal{F}$. The abscissa corresponds to the poloidal angle. $\theta_{p}$. The ordinate is the cosine of the pitchangle along a gyrocenter trajectory. The orbits above the separatrix correspond to co-passing orbits, those below the separatrix correspond to counter-passing orbits. and the elliptical trajectories correspond to trapped orbits. Since kinetic energy is a constant of the unperturbed motion, these orbits are independent of $v_{0}$. The vertical curves in Figure 1. represent the relation $\nu=0$ in this space for a set of values $v_{0}$. The solid curves are those for which $k_{\|}>0$ and the dotted curves are those for which $k_{\|}<0$. The intersections of the gyrocenter trajectories and the curves $\nu=0$ are the resonances corresponding to positions of stationary wave-particle interaction phase. For $k_{i l}=0$, the vertical curves all collapse into a single vertical line passing through the common intersection of the curves. That intersection becomes the turning point or bounce resonance point. For $k_{\|} \neq 0$ the loci of points in the phase space at which wave-particle resonance occurs coincidentally with $\dot{\nu}=0$. tangent resonance. is shown as the remaining curve in Figure 1. This curve, of course, also goes through the turning point resonance. since there is no parallel Doppler shift at that point.

With the previous discussion of resonance broadening in mind, we introduce the change of variables $w(\tau) \rightarrow W\left(\theta_{p}\right)$ and set

$$
B_{0_{q 1}}=\bar{b}_{0} \int d \theta_{p} W\left(\theta_{p}\right) \mathbf{E}_{\mathbf{k}}^{2}\left(\theta_{p}\right)
$$

The quantities $\bar{b}_{0}$ and $W$ are functions of orbit invariants $v_{0}, \theta_{0}$ as well as wave field parameters $\omega, k_{\|}, k_{\perp}$ and wave polarization at resonance. There is in general a certain group of gyrocenter orbits each within its own rangc of resonant interaction at $\theta_{p}$. The particles which populate these orbits all experience the same field spectral energy density $\mathbf{E}_{k}^{2}$ at $\theta_{p}$, though each with its own orbit dependent wave-particle coupling strength $W\left(\theta_{p}\right)$. It is the distribution of population over this orbit group which determines the absorption (or emission) at $\theta_{p}$.

For finite $k_{\|}$there arise situations in which ions can transit through two distinct resonances on a single (quarter) bounce or transit in the magnetic well. Since the absolute interaction phase (the gyrophase at $\theta_{p}$. say) is randomly distributed over all those particles on the same gyrocenter orbit, the ensemble as a whole will either absorb o: emit. but not both. In this sense. the absorption properties of the plasma are non-local. 
The differential power absorption can now be represented as the integral

$$
\frac{d P_{R F}}{d \theta_{\mathrm{p}}}=-2 \pi \int d v_{0} \int d \theta_{0} m v_{0} \sin \theta_{0} \bar{b}_{0} W \mathbf{E}_{\mathbf{k}}^{2} \widehat{\Delta}_{0} \xi_{0}
$$

which is depicted in Figure 2. The depression in the power absorption near the fundamental resonance is a reflection of the fact that for moderate $k_{\|}$. only low energy particles experience vanishingly small doppler shift.

A word about applications: The model as set out in the foregoing is presently in:orporated in the CQL family of codes. The philosophy with which thie present version was developed centers on the mechanism of magnetic decorrelation. Though a charged particle must be in resonance to interact strongly with the exciting RF fields, it is not the case that the magnetic corralation time is the most rational measure of the strength of the interaction in all cases. The magnetic correlation time does, however. represent a persistent uniform underlying structure. In some cases of interest. finite bandwidth wave spectra give rise to wave-particle correlation times shorter than $\tau_{c}$. Models which combine these effects in a rational manner are the subject of current study.

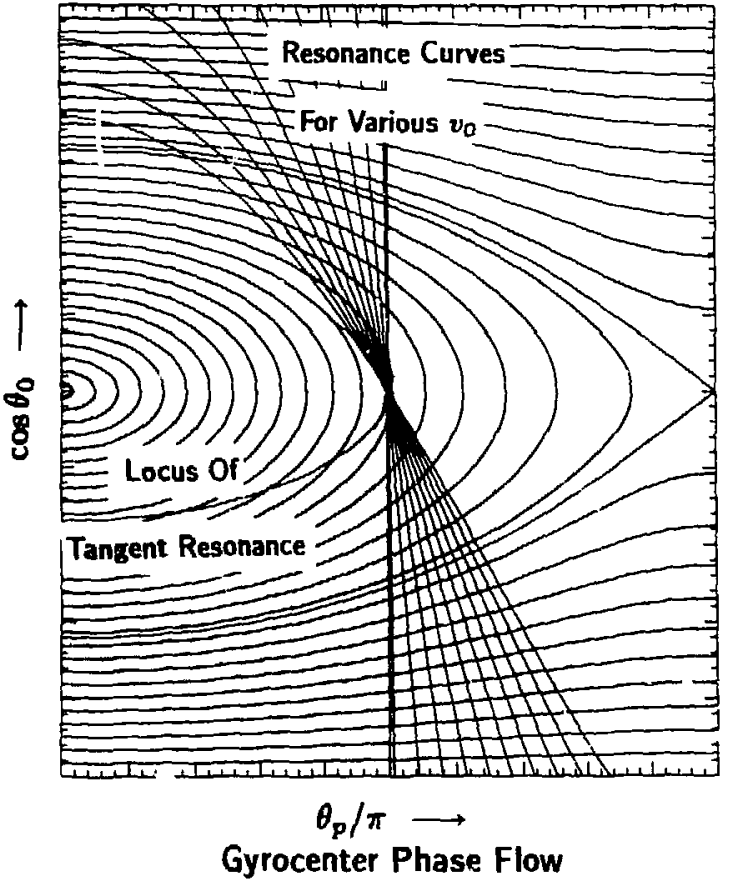

Figure 1.

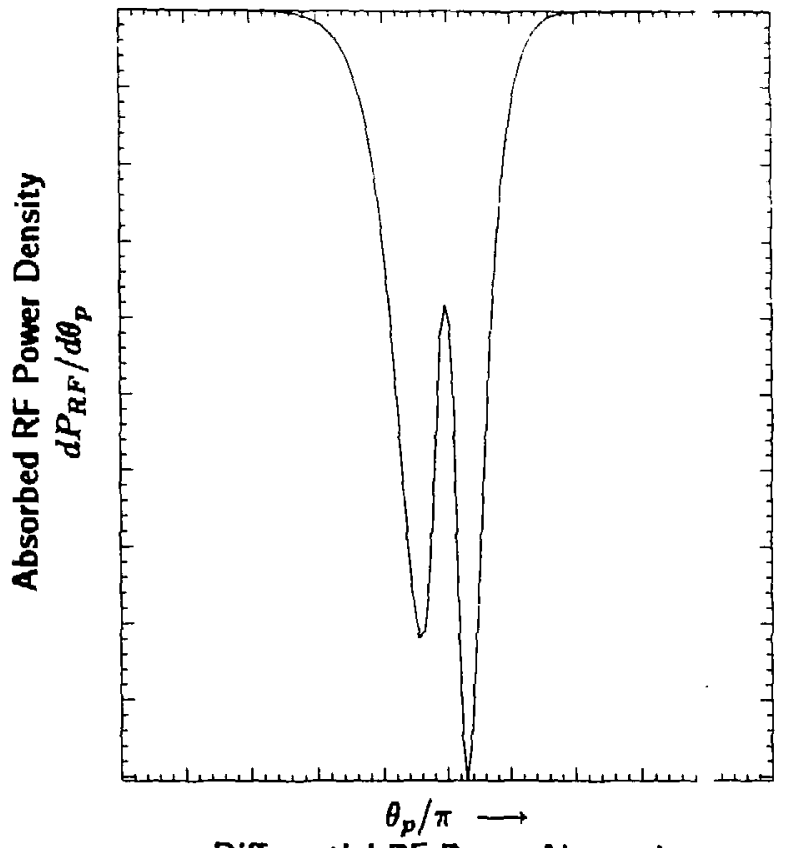

Differential RF Power Absorption Figure 2.

1G.D.Kerbel and M.G.McCoy. Kinetic Theory and Simulation of Multi-Species Plasmas in Tokamaks Excited With ICRF Waves. UCRL-92062. LLNL. (1984). 